\title{
QUALIDADE DE VIDA DO PACIENTE COM INSUFICIÊNCIA RENAL CRÔNICA SUBMETIDO À HEMODIÁLISE
}

Elaine Cristina Negri, Ana Caroline de Lima Sampaio, Ana Cláudia Pereira da Silva, Helora Martines Paulo, Leandro Borsari da Costa, Natalia Fernanda Higa de Souza

Universidade do Oeste Paulista, Curso Enfermagem, Presidente Prudente, São Paulo. e-mail: elainegri@terra.com

\section{RESUMO}

A doença renal crônica é uma afecção multicausal, com elevada taxa de morbidade e mortalidade, que tem a hemodiálise como opção de tratamento mais utilizado. A qualidade de vida é um conceito amplo que envolve um conjunto de bem estar físico, psíquico e social, a qual está associada à noção de felicidade, quando comparados com os aspectos externos. O objetivo da presente pesquisa foi avaliar a qualidade de vida dos pacientes com insuficiência renal crônica submetidos ao tratamento de hemodiálise. A pesquisa foi realizada com 63 pacientes, que responderam a dois questionários, sendo um de caracterização e o instrumento WHOQOL-bref composto por 26 questões. A análise dos dados mostrou que entre os domínios analisados o domínio físico obteve menor pontuação, podendo ser um dos principais fatores que influenciam na qualidade de vida.

Palavras-chave: dialise renal, qualidade de vida, nefropatias, insuficiência renal crônica, dor.

\section{LIFE QUALITY OF THE PATIENT WITH CHRONIC RENAL DISEASE UNDERGOING HEMODIALYSIS}

\begin{abstract}
The chronic kidney disease is multicausal condition with high morbidity and mortality rate, that has hemodialysis as the main option of treatment. Life quality is a large term which includes physical, psychological and social welfare that is related with happiness perception when compared to external aspects. The research goal was to rate the welfare of patients with chronic kidney disease that undergoes hemodialysis treatment. The research was made with 63 patients who filled two formularies: one of them about sample characterization and instrument WHOQOL-bref made by 26 questions. The data analysis shows that among the analyzed areas, the physical domain has got the lowest score which may be one of the main factors that influences the welfare.
\end{abstract}

Keywords: kidney dialysis, welfare, nephropathy, chronic kidney disease, pain.

\section{INTRODUÇÃO}

Doença renal crônica é um dos principais problemas de saúde mundial ${ }^{1}$. Trata-se de uma afeç̧ão multicausal, tratável de várias maneiras, controlável, porém sem cura e com elevada taxa de morbidade e mortalidade ${ }^{2}$.

Caracteriza-se por uma lesão do órgão com perda progressiva e irreversível da função dos néfrons levando a perda da capacidade de manter o equilíbrio metabólico e hidroeletrolítico ${ }^{3-4}$. Estudos revelam que o diagnóstico precoce aliado ao tratamento correto podem prevenir ou retardar as possíveis complicações geradas ${ }^{1,5}$. Os cuidados em relação a doenças como diabetes mellitus, hipertensão arterial, glomerulonefrites são consideradas determinantes para o comprometimento renal e podem diminuir significativamente a incidência de pacientes renais crônicos ${ }^{6}$.

As modalidades para o tratamento são a hemodiálise, diálise peritoneal ambulatorial contínua, diálise peritoneal automatizada e transplante renal. $\mathrm{O}$ que determina a escolha do tipo desses tratamentos é a individualidade de cada paciente, de acordo com o quadro clínico, psico e socioeconômico de cada um² .

A hemodiálise atualmente é o tratamento mais utilizado no mundo, países como o Chile ouve um aumento significativo no número de casos da doença ${ }^{7}$. Dados como esses foram encontrados também no norte do Brasil onde 96,8\% dos participantes realizam hemodiálise e os outros $3,2 \%$ diálise peritoneal ${ }^{8}$. No Brasil segundo a Associação Brasileira de Nefrologia 
(SBN) existem 684 centros no qual 21,9\% estão localizados na região sul do país ${ }^{9}$. O paciente quando se submete ao tratamento hemodiático torna-se dependete de uma máquina, a qual afeta sua autonomia dado o caráter obrigatório, tornando indispensável para manutenção de sua vida $^{10}$.

O procedimento consiste em ligar o paciente a uma maquina na qual fará o papel dos rins, onde o sangue passará pela máquina, ela removerá as substâncias tóxicas e excessos de líquidos, depois o sangue torna ao corpo. As sessões são de aproximadamente 4 horas, em média três vezes por semana, dependendo da necessidade do indivíduo ${ }^{11}$. Durante o tratamento podem apresentar algumas alterações como fraqueza muscular, cãibras, pele seca, dormência, estresse entre outros, levando o paciente a apresentar dificuldade de realizar certas atividades de seu cotidiano. Alterações como essas aumentam a insegurança levando ao consequente sedentarismo, mesmo estudo comprova a eficácia de exercícios fiscos na melhoria da capacidade funcional e além de melhora na qualidade de vida ${ }^{12}$.

Segundo Almeida et al. ${ }^{13}$ qualidade de vida considera algumas variáveis, sendo essas bem estar físico, psíquico e social, associadas aos fatores externos, considerando questões imensuráveis como por exemplo a felicidade. 0 Grupo de Qualidade de Vida da Organização Mundial de Saúde (OMS) inclui na concepção de qualidade de vida, seis domínios: saúde física, estado psicológico, níveis de independência, relacionamento social, características ambientais e padrão espiritual, nos quais cada indivíduo pode enfatizar para si de acordo com seu contexto um determinado domínio ${ }^{14}$.

De acordo com Ferreira e Silva Filho ${ }^{15}$ a hemodiálise é responsável por alterações significativas na qualidade de vida dos pacientes renais crônicos. Guedes e Guedes ${ }^{16}$ mostram em seus estudos que a doença renal crônica está entre as de maior impacto na qualidade de vida do paciente, decorrente de vários fatores, como o esquema terapêutico rigoroso, o convívio com a doença incurável e a dependência de uma máquina e limitações provenientes do processo terapêutico.

Diante disto, justifica-se a importância de se conhecer as necessidades humanas afetadas, e identificar as necessidades e expectativas dos portadores de doença renal crônica, submetidos à hemodiálise, a fim de contribuir na busca da melhoria da qualidade de vida do paciente com doença renal crônica.

O objetivo da presente pesquisa foi avaliar a qualidade de vida dos pacientes com insuficiência renal crônica, submetidos ao tratamento de hemodiálise.

\section{MÉTODOS}

Estudo de natureza descritiva, quantitativa, com coleta de dados realizada com 63 indivíduos com doença renal crônica submetido a tratamento hemodialítico em um serviço de hemodiálise na cidade de Presidente Prudente - SP. A coleta de dados foi realizada através da aplicação de formulários, após aprovação do Comitê de Ética em Pesquisa da Universidade do Oeste Paulista (protocolo no 1095/2012) e mediante a concordância e assinatura do termo de consentimento livre e esclarecido dos participantes.

Foram utilizados dois instrumentos, sendo um instrumento composto por questões dirigidas abordando os aspectos socioeconômicos, sexo, idade, estado conjugal, grau de instrução, complicações durante a sessão de hemodiálise, sendo estas elaboradas pelos autores do trabalho com identificação anônima dos participantes do estudo. $O$ outro instrumento utilizado para analisar a qualidade de vida, constitui o questionário WHOQOL-bref ${ }^{17}$ (Word Health Organization Quality of Life - abreviado) composto por 26 questões, dividido em quatro domínios: físicos, psicológico, social e meio ambiente.

As respostas para todas as questões do WHOQOL-bref são obtidas através de uma escala de respostas: intensidade, capacidade, frequência e avaliação, todas graduadas em cinco níveis 1 5 , além de duas questões sobre qualidade de vida geral calculadas em conjunto para gerar um único escore independente dos escores dos domínios, denominada Overall ou "qualidade de vida geral". Para o cálculo dos escores dos domínios os índices das facetas componentes resumem os domínios aos quais pertencem. Tantos os domínios quanto à qualidade de vida geral são medidos em direção positiva, ou seja, escores mais altos denotam melhor qualidade de vida. A partir dai foi realizada uma regressão multipla, para analisar quais dominios explicam as variações de cada questão, porém para que cada um dos testes assumissem $95 \%$ de confiança o valor de $p$ deveria ser menor igual a 0,05. 
A tabulação dos dados obtidos foi realizada no MS Excel 2007 e as análises estatísticas no SAS versão 9.1.3.

\section{RESULTADOS}

Do total dos 63 sujeitos que participaram da pesquisa $53,97 \%$ (34) eram do sexo masculino, 46,03\% (29) do sexo feminino. A faixa etária que apresentou maior percentual de participantes foi de 51 a 64 anos com 41,09\% (23). Em relação à situação conjugal $68,84 \%$ (44) eram casados. Quanto à escolaridade a maioria $33,33 \%$ (21) referiu ensino fundamental incompleto, seguido de ensino médio completo $30,16 \%$ (19).

Tinham a doença renal a 5 anos ou mais $44,44 \%$ (28). Quando questionados sobre quanto tempo realizavam a hemodiálise $28,57 \%$ (18) referiram realizar este procedimento a 5 anos ou mais. Quanto ao número de sessões de hemodiálise $96,83 \%$ (61) refere realizar três vezes por semana; $82,54 \%$ (52) dos sujeitos referem ter apresentado algum tipo de complicações durante as sessões de hemodiálise e 79,37\% (50) referem apresentar outra doença.

A Tabela 1 ilustra a média específica obtida em cada domínio analisado pela escala de aferição de qualidade de vida WHOQOL-bref.
Em relação ao questionário $\mathrm{WHOQOL-}$ bref, o domínio 1 - físico que compreende a dor, o desconforto e energia, a fadiga, o sono e o repouso, o que mais influencia a qualidade de vida do sujeito submetido à hemodiálise obteve a menor pontuação, comparativamente aos outros domínios pesquisados. Domínio 4 - meio ambiente, domínio 2 - psicológico e o domínio 3 relações sociais, vieram em sequencia com escores maiores. Quanto ao escore geral - overall de QV geral para a população estudada apresentou média de 78,27 (Tabela 1).

Foi realizada uma regressão múltipla para analisar o quanto cada domínio explica cada questão, onde o valor de significância dos testes deve ser $p$ inferior igual a 0,05 (com intervalo de confiança de $95 \%$ ).

A faceta 18 que corresponde a capacidade de trabalho, pode ser explicada pelos domínios 1 e 2, onde observamos no domínio 1 o valor de $\mathrm{p}=0,001$ e no domínio 2 valor de $\mathrm{p}=0,01$, demonstrando que a satisfação com a capacidade de trabalho pode afetar não só a qualidade de vida física como a psicológica da pessoa.

Tabela 1. Análise dos domínios WHOQOL-bref.

\begin{tabular}{l|c|c|c}
\hline Domínio & Média \pm DP & Mínimo & Máximo \\
\hline 1. Físico & $59,52 \pm 18,76$ & 14,28 & 100 \\
2. Psicológico & $69,11 \pm 15,44$ & 29,17 & 100 \\
3. Relações Sociais & $75,53 \pm 16,59$ & 25,00 & 100 \\
4. Meio ambiente & $68,60 \pm 13,97$ & 34,38 & 100 \\
Overall - domínio geral & $78,27 \pm 19,54$ & 18,75 & 106,25 \\
\hline
\end{tabular}

\section{DISCUSSÃO}

O termo qualidade de vida (QV) tem sido muito usado como um conceito necessário na prática dos cuidados e pesquisa em saúde. A Organização Mundial de Saúde $(\mathrm{OMS})^{18}$, em 1948, definiu saúde como não apenas ausência de doença ou enfermidade, mas também a presença de bem-estar físico, mental e social. Portanto nas pesquisas alguns dados podem apresentar maior relevância que outros, ou seja, a noção de qualidade de vida pode variar de acordo o grau de importância dada a determinados aspectos.

$\mathrm{Na}$ pesquisa os resultados mostraram a predomínio de pessoas do sexo masculino que hemodializam na respectiva unidade, indo de encontro com outras pesquisas ${ }^{5,19,20}$. O predomínio do público masculino justifica-se pelo fato da maioria dos homens não passarem por consultas periódicas e cuidarem menos da saúde se comparado às mulheres. Quando apresentam alguma patologia, a mesma muitas vezes está em estado de evolução mais comprometedor, sendo necessárias intervenções mais complexas.

O fato da maioria dos indivíduos serem casados segundo Pivatto e $\mathrm{Abreu}^{20}$, torna-se importante, pois a presença de um companheiro durante o processo de tratamento auxilia nas limitações, apoiando emocionalmente através do vínculo familiar. 
Além do companheiro(a) o conhecimento também pode interferir positiva ou negativamente na adesão do tratamento e compreensão das orientações realizadas. Sendo de fundamental importância que o enfermeiro aborde estes indivíduos com informações claras, precisas, com linguagem acessível e adequada escolaridade dos mesmos.

O tempo de hemodiálise influencia em aspectos importantes, pois estudo realizado em um hospital de Belém com pacientes em programa de hemodiálise mostrou que indivíduos com maior tempo de tratamento apresentam melhor capacidade física, mental e social de enfrentar a doença, afirmando ainda que o passar do tempo faz com que os pacientes com doença renal crônica se ajustem melhor à sua doença e através de sentimentos de conformismo e de aceitação do seu estado de saúde. $^{21}$ No entanto Takemoto et al. ${ }^{22}$ mencionam que, quanto maior tempo de tratamento, maior as chances de interferência nos aspectos emocionais.

Em relação às complicações que os pacientes apresentam durante as sessões de hemodiálise, não foi evidenciado na literatura estudos que demonstram a influência das complicações durante as sessões de hemodiálise na QV dos pacientes. Porém, ao cruzarmos os dados das variáveis como WHOQOL-bref, esta apresentou valores estatisticamente significante no domínio geral demonstrando que ter complicações durante as sessões de hemodiálise pode interferir na qualidade de vida geral dos indivíduos submetidos ao tratamento hemodialitico. Além de gerar insegurança nos pacientes, podendo diminuir a adesão terapêutica.

$\mathrm{Na}$ analise dos domínios a maior influência na QV foi apresentada no domínio físico que obteve menor pontuação de 59,52 corroborando com os estudos de Ferreira e Silva Filho $^{15}$. Dados como este sugerem que com o passar do tempo e o surgimento de complicações aliado a sobre carga emocional levam o indivíduo a uma maior dependência, onde acaba por privar-se de realizar atividades rotineiras.

A satisfação com a capacidade de trabalho foi significante entre as facetas e justificadas nos domínios físico e psicológico, ou seja, quando o indivíduo insatisfeito com sua capacidade de trabalho, acaba por privar-se de outras atividades físicas julgando ser incapaz de realizá-las, dessa forma cria uma limitação não apenas física como psicológica.

\section{CONCLUSÃO}

$O$ individuo com insuficiencia renal crônica pode ter sua qualidade de vida afetada por diversos fatores, porém o grau de influência depende da importancia dada pelo induviduo a cada aspecto envolvido.

Este estudo demonstrou que o domínio físico influencia a qualidade de vida dos pacientes com insuficiência renal crônica submetidos à hemodiálise, ocasionando mudanças no estilo de vida do paciente, exigindo muitas vezes adaptações nas atividades cotidianas. Neste contexto o tratamento imposto é um dos principais motivos que levam o paciente a insegurança sobre sua vida e futuro, pois as limitações decorrentes podem gerar sentimentos de incapacidade tanto física quanto psicológica, que de certa forma levam a incertezas e aumenta o índice de adoecimento. Este estudo pode subsidiar os profissionais de saúde para que possam propor ações que previna o comprometimento das atividades cotidianas desses pacientes.

\section{CONFLITO DE INTERESSE}

Os autores declaram não haver qualquer potencial de conflito de interesse que possa interferir na imparcialidade deste trabalho científico.

\section{REFERÊNCIAS}

1. Bastos MG, Bregman R, Kirsztajn GM. Doença renal crônica: frequente e grave, mas também prevenível e tratável. Rev Assoc Med Bras. 2010;56(2):248-53. DOI: http://dx.doi.org/10.1590/S0104-

\section{8}

2. Frazão CMFQ, Ramos VP, Lira ALBC. Qualidade de vida de pacientes submetidos à hemodiálise. Rev Enferm. UERJ. 2011;19(4):577-82.

3. Cunha MS, Andrade V, Guedes CAV, Meneghetti $\mathrm{CHZ}$, Aguiar AP, Cardoso AL. Avaliação da capacidade funcional e da qualidade de vida em pacientes renais crônicos submetidos a tratamento hemodialítico. Fisioter Pesqui. 2009;16(2):155-60. DOI: http://dx.doi.org/10.1590/S1809-

\section{1}

4. Mortari DM, Menta M, Scapini KB, Rockembach CWF, Duarte A, Leguisamo CP. Qualidade de vida de indivíduos com doença renal crônica terminal submetidos à hemodiálise. Sci Med. 2010;20(2):15660. 
5. Higa K, Kost MT, Soares DM, Morais MC, Polins BRG. Qualidade de vida de pacientes portadores de insuficiência renal crônica em tratamento de hemodiálise. Acta Paul Enferm. 2008;21(spe):203-6. DOI: http://dx.doi.org/10.1590/S010321002008000500012

6. Marchesan M. Efeitos de um programa de exercícios físicos sobre a qualidade de vida e a reabilitação física de pacientes submetidos à hemodiálise [dissertação]. Pelotas (RS): Universidade Federal de Pelotas; 2010.

7. Guerra-Guerrero V, Sanhueza-Alvarado O, CáceresEspina M. Qualidade de vida de pessoas em hemodiálise crônica: relação com variáveis sociodemográficas, médico-clínicas e de laboratório. Rev Latino-Am Enfermagem. 2012;20(5):838-46. DOI: http://dx.doi.org/10.1590/S0104-

\section{4}

8. Mendonça AEO, Torres GV, Salvetti MG, Alchieri JC, Costa IKF. Mudanças na qualidade de vida após transplante renal e fatores relacionados. Acta Paul Enferm. 2014;27(3):287-92. DOI: http://dx.doi.org/10.1590/1982-0194201400048

9. Silva AS, Silveira RS, Fernandes GFM, Lunardi VL, Backes VMS. Percepções e mudanças na qualidade de vida de pacientes submetidos à hemodiálise. Rev Bras Enferm. 2011;64(5):839-44.

DOI:

http://dx.doi.org/10.1590/S0034-

\section{6}

10. Medeiros AJS, Medeiros EMD. A assistência de enfermagem prestada no tratamento hemodialítico promovido junto ao portador de insuficiência renal crônica - Uma revisão de literatura. REBES. 2013;3(2):13-17.

11. Marchesan M, Krug RR, Ritter AJ, Romiti JC, Daltrozo MA, Krug MR et al. Composição corporal de pacientes com insuficiência renal crônica. Rev Digital Buenos Aires. 2009;14:(135).

12. Costa PB, Vasconcelos KFS, Tassitano RM. Qualidade de vida: pacientes com insuficiência renal crônica no município de Caruaru, PE. Fisioter Mov. 2010;23(3):461-71.

DOI:

http://dx.doi.org/10.1590/S0103-

\section{3}

13. Almeida MAB, Gutierrez GL, Marques R. Qualidade de vida: definição, conceitos e interfaces com outras áreas, de pesquisa. Prefácio do professor Luiz Gonzaga Godoi Trigo. - São Paulo: Escola de Artes, Ciências e Humanidades - EACH/USP, 2012.

14. Ramos-Dias JC, Libardi MC, Zillo CM, Igarashi $\mathrm{MH}$, Senger $\mathrm{MH}$. Qualidade de vida em cem alunos do curso de Medicina de Sorocaba - PUC/SP. Rev Bras Educ Med. 2010;34(1):116-23. DOI: http://dx.doi.org/10.1590/S0100-

\section{4}

15. Ferreira RC, Silva Filho CR. A qualidade de vida dos pacientes renais crônicos em hemodiálise na região de Marília, São Paulo. J Bras Nefrol. 2011;33(2):129-35.
DOI: http://dx.doi.org/10.1590/S010128002011000200003

16. Guedes KD, Guedes HM. Qualidade de vida do paciente portador de insuficiência renal crônica. Rev Ciên Saúde. 2012;5(1):48-53.

17. The WHOQOL Group. The Word Health Organization Quality of Life Assessment (WHOQOL): Position paper from the Health Organization. Soc Sci Med. 1995;41(10):1403-9.

18. World Health Organization. Preamble to the Constitution of the World Health Organization as adopted by the International Health Conference, New York, 19-22 June, 1946; signed on 22 July 1946 by the representatives of 61 States (Official Records of the World Health Organization, no. 2, p. 100) and entered into force on 7 April 1948 [acesso em 10 mar 2016]. Disponível em: http://www.who.int/about/definition/en/print.html 19. Patat, CL, Stumm EMF, Kirchner RM, Guido LA, Barbosa DA. Análise da qualidade de vida de usuários em hemodiálise. Enfermería Global. 2012;11(27):5465.

20. Pivatto DR, Abreu IS. Principais causas de hospitalização de pacientes em hemodiálise no município de Guarapuava, Paraná, Brasil. Rev Gaúcha Enferm. 2010;31(3):515-20. DOI: http://dx.doi.org/10.1590/S1983-

14472010000300015

21. Silveira CB, Pantoja IKOR, Silva ARM, Azevedo RN, Sá NB, Turiel MGP, et al. Qualidade de vida de pacientes em hemodiálise em um hospital público de Belém - Pará. J Bras Nefrol. 2010;32(1):39-44. DOI: http://dx.doi.org/10.1590/S0101-

28002010000100008

22. Takemoto AY, Okubo P, Bedendo J, Carreira L. Avaliação da qualidade de vida em idosos submetidos ao tratamento hemodialítico. Rev Gaúcha Enferm. 2011;32(2):256-62. DOI: http://dx.doi.org/10.1590/S1983$\underline{14472011000200007}$

Recebido para publicação em 17/08/2015

Revisado em 18/08/2015

Aceito em 21/08/2015 